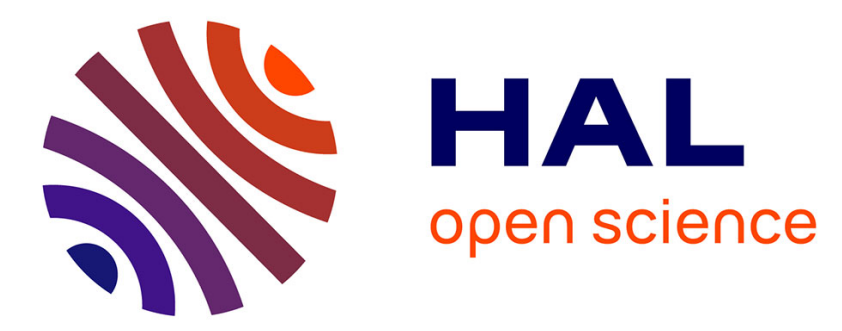

\title{
Modeling wear for heterogeneous bi-phasic materials using discrete elements approach
}

Matthieu Champagne, Mathieu Renouf, Yves Berthier

\section{To cite this version:}

Matthieu Champagne, Mathieu Renouf, Yves Berthier. Modeling wear for heterogeneous bi-phasic materials using discrete elements approach. Journal of Tribology, 2014, 136 (2), pp.021603. 10.1115/1.4026053 . hal-01003460

\section{HAL Id: hal-01003460 \\ https://hal.science/hal-01003460}

Submitted on 16 Jan 2017

HAL is a multi-disciplinary open access archive for the deposit and dissemination of scientific research documents, whether they are published or not. The documents may come from teaching and research institutions in France or abroad, or from public or private research centers.
L'archive ouverte pluridisciplinaire HAL, est destinée au dépôt et à la diffusion de documents scientifiques de niveau recherche, publiés ou non, émanant des établissements d'enseignement et de recherche français ou étrangers, des laboratoires publics ou privés. 


\title{
Modeling wear for heterogeneous bi-phasic materials using discrete elements approach
}

\author{
Matthieu Champagne \\ PhD Student \\ CNRS LAMCOS - UMR5259 \\ Université de Lyon \\ Villeurbanne, France, F-69621 \\ Yves Berthier \\ Research Professor \\ CNRS LAMCOS - UMR5259 \\ Université de Lyon \\ Villeurbanne, France, F-69621 \\ Email: yves.berthier@insa-lyon.fr
}

Email: matthieu.champagne@insa-Iyon.fiEmail: mathieu.renouf@univ-montp2.fr

\begin{abstract}
A proper understanding of the processes of friction and wear can only be reached through a detailed study of the contact interface. Empirical laws, such as Archard's, are often used in numerical models. They give good results over a limited range of conditions when their coefficients are correctly set, but they cannot be predicted: any significant change of conditions requires a new set of experimental coefficients. In this paper, a new method, the use of Discrete Element Models (DEM), is proposed in order to tend to predictable models. As an example, a generic biphasic friction material is modelled, of the type used in aeronautical or automotive brake systems. Micro-scale models are built in order to study material damage and wear under tribological stress. The models show what could be achieved by these numerical methods in tribological studies, and how they can reproduce the behavior and mechanisms seen with real-life friction materials without any empirical law or parameter.
\end{abstract}

\section{Tribological context}

Understanding the wear process is an essential part of the study of dry friction problems. Even if friction and wear are common and every-day phenomena, they are nonetheless complex multi-scale and multi-physical mechanisms [1]. Friction and wear depend on parameters such as material properties, surface characteristics, stress cycles, mechanisms, etc. All these parameters are strongly connected and cannot easily be studied separately. Moreover, no single model can be used to describe a friction and wear process at all scales, whilst taking account of all these parameters. Being of industrial and economic importance, wear has led to many studies to try to understand the wear process and to predict its effects. For this reason, both experimental and numerical studies are mandatory: experimental studies are limited by cost, inner contact visibility, complex instrumentation, etc. and require the support of a numerical model to be useful; but the model needs to be fed with experimental results, measurements and observations.

Numerical studies of friction and wear found in the literature often use theoretical laws based on simple and general hypotheses represented through different parameters. Their goal is to offer a quantitative prediction of wear "magnitude" which depends on these parameters. These laws are supposed to be adaptable to any system under study, accounting for normal contact force or sliding distance. For example, Archard's well known law [2] is widely used in numerical models to predict the worn volume obtained under given conditions (normal force and sliding distance). This law has been cleverly written but uses a constant coefficient, which must be chosen to adapt the law to the phenomenon being studied. Other laws such as Rhee's [3] and Barwell's [4] are made to fit experimental results so as to predict the long term wear effect. Most of the time, the final result is given as the worn volume. Industrial interests encourage the use of such laws, emphasising the needs of long-term fatigue studies, and taking the "wear magnitude" obtained under given conditions and during a given time as the key output of the models. 
From a numerical point of view, different methods can be found in the literature applied to these studies, based on two main approaches. The first is applied at the macro scale and uses a wear law or criterion as an input to the model, not always related to the problem being studied, to compute a worn volume or a worn surface. The second is applied at the micro scale and studies the wear mechanism itself, trying to reproduce experimental phenomena explicitly and giving wear parameters as an output.

In the first approach, the Finite Element Method (FEM) is probably the most widely used and popular method, particularly at macro scale. For example, Chongyi et al [5] use such a method to model the wheel / rail contact, using local results (such as normal pressure and sliding velocity) in an Archard-like law as wear model, to predict worn material volume after each time iteration. This volume is then eliminated from the existing material and both geometry and meshing are corrected. In other work, FEM models are used to predict wear using an empirical law [6] where an abrasive wear coefficient, obtained from experiments, can be found. This approach can also be found in [7-9]. In the work of Gallego et al [10,11], the local results of a higher scale FEM model are used in a semi-analytical contact solver. Archard's law is then used to compute wear distribution and the new worn surface is updated in the FEM model. The advantage of such a model is that it is fast enough to perform cyclic wear computation and fatigue life studies.

Although useful to some extent, these models are not comprehensive: they simply create a fit to an existing process under precise conditions (for example temperature). The wear coefficient used in Archard's law reveals the empirical aspect of this law, and using it in inappropriate conditions can lead to incoherent results. As regards wear modeling, the main drawback of such models is that wear is only considered as a material removal process. The third body and wear particles are not taken into account, leading some authors to puzzle over the decrease in wear volume seen in experiments but not in their models [12], while this in fact could be explained by a third body layer accommodation mechanism [13]. Others point out that third body studies are needed as a perspective for their models [8]. Sometimes, due to this lack of comprehensivity, predictive models using Archard's laws need a limit condition in worn volume computation, and this parameter is often arbitrary (for example a maximum allowable wear depth in [5]).

In contrast to previous models, micro scale numerical models can provide specific laws or parameters directly deduced from modelled wear mechanisms, and thus can be an alternative way to feed higher scale models. The continuum mechanics (FEM model and Linear Fracture Mechanics [14], the continuous Granular Kinetic Lubrication model [15]) have been used in models with such objectives, but continuum methods quickly show their limits when modeling a discontinuous environment such as the third body layer. Discrete mechanics methods can then be considered: the goal is no longer to use a global or local wear law, but explicitly to study material degradation, wear particle creation, and particle life within the contact interface (i.e. the role of the third body layer). For example, some authors [16-18] use the movable Cellular Automata method, a very simplified way of modeling a granular flow (as the third body can be); but it lacks some important aspects for studying wear process (such as thermochemical aspects). However, the Discrete Element Method (DEM) appears to be very promising for studying material degradation and third body flows in a contact. Under simple shear [19], where a wear law is obtained, the authors find that Archard's law is applicable in a limiting case where all wear particles are expelled from the contact (which is not always the case!), and under fretting-like conditions [20], where these models proved to be a useful and powerful tool for investigating the wear process. These models use the conceptual approach of the tribological triplet $[1,21]$ and the tribological circuit model [13], which are tools for understanding and interpreting wear processes. They provide descriptive schemes which can lead to comprehensive models. As a consequence, comprehensive micro scale models which take third body into account so as to understand local wear phenomena with wear laws and parameters as outputs, are being steadily developed. Yet at the micro scale, no study has yet really considered the material heterogeneity of the third body layer. When frictional materials are considered, such as brake pads [22] or Carbon/Carbon composites [23], shearing a homogeneous material is no longer representative, and local improvements are needed.

In the present paper, it is proposed to use the DEM method in order to investigate the friction and wear process of a biphasic composite under tribological conditions (pressure and shear velocity). As a first step, a simplified material composed of a matrix and vertical inclusions is considered. The goal of these studies is to model first body degradation (cracks and damage) at the micro scale, wear particle creation and flow, and the influence of inclusions on the process. To do so, Cohesive Zone Models (CZM) are implemented in a DEM model. The influence of different parameters is studied (pressure, CZM parameters), and finally a method to obtain parameters representative of wear phenomena and friction is given. Section 2 presents the numerical model used to describe the bi-phasic composite. Section 3 presents the numerical results and section 4 proposes a synthesis and comparison with experimental results from the literature.

\section{Numerical framework \\ 2.1 NSCD overview}

Several discrete element methods have been used for tribological purposes [19, 24, 25] each based on a different formalism. The one used in the present work is the Non-Smooth Contact Dynamics approach (NSCD), initially developed by Moreau and Jean [26,27]. Often used in the context of granular material investigation [28], it has also been used several 
times in tribological applications [29-31].

The NSCD approach uses an implicit time discretized formulation of the equation of motion written for a collection of particles. Since the number of contacts is higher than the number of particles (dense particle assemblies), the interaction between particles is not considered at the scale of each individual particle but at each contact scale.

To describe the evolution of a collection of particles, the equation of dynamics can be written as:

$$
\mathbb{M} \ddot{\mathbf{q}}+\mathbf{F}^{\text {int }}(t, \mathbf{q}, \dot{\mathbf{q}})=\mathbf{F}^{e x t}(t, \mathbf{q}, \dot{\mathbf{q}})+\mathbf{R},
$$

where $\mathbb{M}$ represents the mass matrix, $\mathbf{F}^{\text {int }}(t, \mathbf{q}, \dot{\mathbf{q}})$ are the internal force and the nonlinear inertia terms, $\mathbf{F}^{\text {ext }}(t, \mathbf{q}, \dot{\mathbf{q}})$ the external forces, and $\mathbf{R}$ the contact forces. Vector $\mathbf{q}$ represents the vector of generalized degrees of freedom while $\dot{\mathbf{q}}$ and $\ddot{\mathbf{q}}$ denote the generalized velocity and acceleration vectors respectively.

To transfer information from the contact frame (local level) to the body frame (global level) (cf. Fig. 1), two linear mappings, $\mathbb{H}$ and its transpose $\mathbb{H}^{*}$, are used.

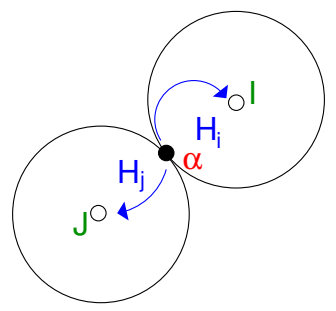

Fig. 1. Representation of the linear mappings of the contact frame (local level) onto body frame (global level). The black dot represents a contact point connected to the local frame while the white dots represent the centre of the mass of particles connected to the contact point by linear mapping $\mathbb{H}$

So it is possible to relate the local contact force vector $\mathbf{r}$ and the local relative velocity vector $\mathbf{u}$ to the global body contact force vector $\mathbf{R}$ and the body velocity vector $\dot{\mathbf{q}}$ thus:

$$
\left\{\begin{array}{l}
\mathbf{R}=\mathbb{H} \mathbf{r} \\
\mathbf{u}=\mathbb{H}^{*} \dot{\mathbf{q}}
\end{array}\right.
$$

Both mappings depend on local information such as the local frame defined at each contact point and the network connectivity of each contact (cf. Fig. 1).

By using a system (2) and a $\theta$ time integrator scheme [27], it is possible to reformulate equation (1) in the contact frame. This allows to us write, at each time step, the system (3) containing the equations of motion:

$$
\left\{\begin{array}{l}
\mathbb{W} \mathbf{r}-\mathbf{u}=\mathbf{b} \\
\operatorname{law}_{\alpha}\left[\mathbf{u}_{\alpha}, \mathbf{r}_{\alpha}\right]=\text { true }, \alpha=1, n_{c}
\end{array}\right.
$$

Where the matrix $\mathbb{W}$ is the Delassus operator, containing local information (such as local frames and contact points) allowing it to be used at the particle scale. The vectors $\mathbf{u}$ and $\mathbf{r}$ are respectively the vectors containing the relative velocity and the mean contact impulse for all the contact points of all particles. $\mathbf{b}$ represents the free relative velocity calculated by taking into account the external forces only. The $\theta$-method is used for time discretization and a classical non-linear GaussSeidel algorithm is used to solve the system (3) [27]. The approach benefits from a parallel version to ensure reasonable CPU times [32].

\subsection{Interaction law for tribological purposes}

A key point of the system (3) is the definition of the law connecting the unknown $\mathbf{u}$ and $\mathbf{r}$. Indeed, though unilateral contact with Coulomb friction may be used to model a granular material, it is not sufficient for tribological purposes, especially when the discretely packed particles initially represent a continuum, from which particles may be detached by tribological 
stress then subsequently evolve through interaction between themselves and with the continuum. Consequently, two interaction laws are used: firstly, a cohesive zone model (CZM) [33,34] is used to model the continuum with discrete packing, from which particles may be detached according to a damage criterion (first body law); and secondly, a unilateral cohesive contact law which models the interactions between detached particles as well as interactions between detached particles and the continuum (third-body law).

\subsubsection{First body law}

In the literature, CZM $[33,34]$ are usually combined with finite elements to model crack evolution in materials such as concrete [35], zircaloy [36], etc. Such a model allows multi-cracking studies where no pre-crack is needed, nor crack path predicted. Here the method is implemented in a DEM model, which leads to an innovative way of studying the wear mechanism. The tribological history of the first body (damage accumulation) and its degradation allow the creation of third body and its life in the contact to be modelled. The present model, used to describe the interaction between 1 st body particles, was first proposed by Raous et al [37] and is based on five parameters: $C_{n}$ and $C_{t}$, the normal and tangential stiffness respectively; $w$, the de-cohesion energy; $b$, the viscosity associated with the evolution of inter-particle adhesion and $\mu$ the local friction coefficient. The intensity of the adhesion of the interaction is characterized in this model by the internal variable $\beta$, introduced by Frémond [38]. It takes values between 0 and 1 ( 0 is no adhesion and 1 is perfect adhesion). A damageable stiffness is used for the interface, depending on $\beta$, to ensure good continuity between contact conditions throughout the process.

$$
\frac{\partial \beta}{\partial t}=-\frac{1}{b}\left(w-\beta\left(C_{n} u_{n}^{2}+C_{t} u_{t}^{2}\right)\right)^{-}
$$

where $(.)^{-}$denotes the negative part. For the purposes of the present work, inter-particle friction is zero $(\mu=0)$, as is viscosity $(b=0)$. The initial interactions are perfectly intact $(\beta=1)$ and normal and tangential stiffness are considered equal. This means that the local model is defined by two parameters, i.e. $C_{n}$ and $w$.

(a)

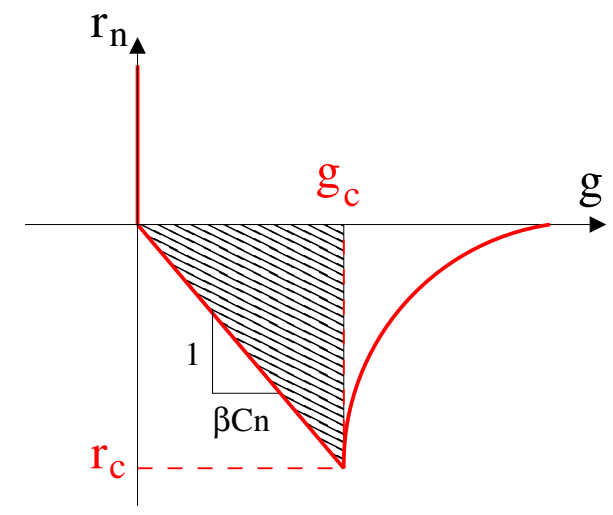

(b)

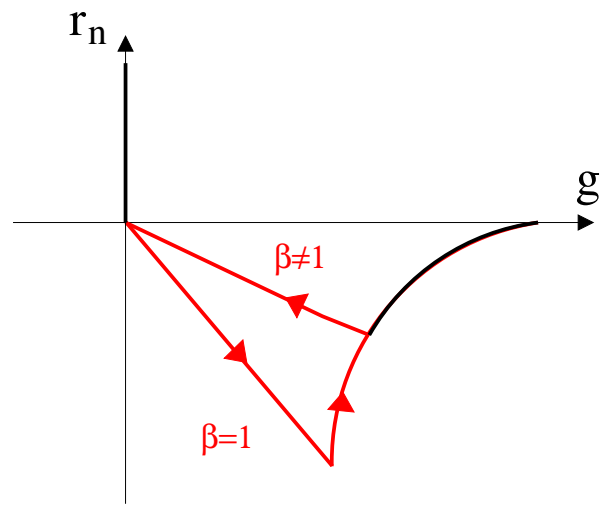

Fig. 2. Cohesive Zone Model force profile ; (a) elastic range definition and (b) beta value.

Figure 2 gives an example of a typical CZM graph. The maximum traction force $r_{c}$ is equal to $\sqrt{w C_{n}}$ while the corresponding distance defining the elastic range (without damage), $g_{c}$, is equal to $\sqrt{w / C_{n}}$. Note that when such a law is used in discrete element assemblies, care is needed during the contact detection process. Indeed, to avoid overlap between particles, an alert distance is used to anticipate a potential contact. Usually, this distance should not be too large so as to minimize the number of contacts (active or not). With the presence of CZM, the alert distance should not be so small as to truncate the CZM graph, which would lead to numerical overestimation of the dissipation of energy.

\subsubsection{Third body law}

For the purposes of this work, a non-smooth cohesive contact law is used. It may be seen as the non-smooth extension of a force which derives from a Lennard-Jones potential. Two parameters are considered: a cohesive force $\gamma$ that represents 
a constant force in opposition to body detachment and a distance $d_{w}$ that defines the attraction area of each body. If body attraction areas do not overlap, then the contact status is non contact $\left(r_{n}=0\right.$ and $\left.g>d_{w}\right)$.Otherwise, the following linear complementarity problem is solved:

$$
g \geq 0, \quad r_{n}+\gamma \geq 0, \quad g \cdot\left(r_{n}+\gamma\right)=0 .
$$

Therefore the contact status is cohesive when $\gamma<r_{n}<0$ and $g \leq d_{w}$. Finally the contact status is stick when the gap vanishes $(g=0)$ and $r_{n} \geq 0$. When $\gamma=0$ then $d_{w}=0$ and cohesion vanishes. In this case the description of the contact is the classical Signorini condition used to simulate dry systems [27].

\subsection{Model}

Figure 3 represents a typical sample of the biphasic material used in this work: a uniform matrix and several vertical inclusions. The inclusions represent 10\% of the whole surface. CZM parameters are chosen according to the properties of the particles in interaction (matrix-matrix; matrix-inclusion; inclusion-inclusion). The cohesion forces used in Equation (5) have been chosen with regard to the dimensionless parameter $\eta[39]$ defined as,

$$
\eta=\frac{\gamma}{P d}
$$

where $P$ is the normal pressure and $d$ the particle diameter. Consequently, if $P$ and $d$ are constant, the evolution of eta is directly related to the evolution of $\gamma$. In shear simulations of cohesive granular flows [39], the measured global friction coefficients exhibit a plateau for values of $\eta$ less than 1 and a large increase above this value. The different values of $\gamma$ have been chosen here to describe the plateau, except for the higher value of $\gamma\left(\right.$ i.e. $\gamma=10^{-2} N$ ) which corresponds to a value of $\eta$ equal to 1.33 .

The value of CZM stiffness has been chosen to ensure that the global stiffness of the material corresponds to a range of values close to those of friction materials. The matrix stiffness is less than that of the inclusions (with sufficient contrast), and the order of magnitude of these parameters is adjusted according to model size, particle size and the external forces applied. The upper boundary, where normal force is applied, is connected to the matrix/inclusions via CZM interactions (and so it is part of the upper 1st body) while the lower boundary, where shear velocity is applied, is connected to the sample through the unilateral cohesive law (so that it represents the lower 1st body - see Fig. 3). The degrees of freedom of upper and lower boundaries (other than those where normal force and shear velocity are applied) are blocked.

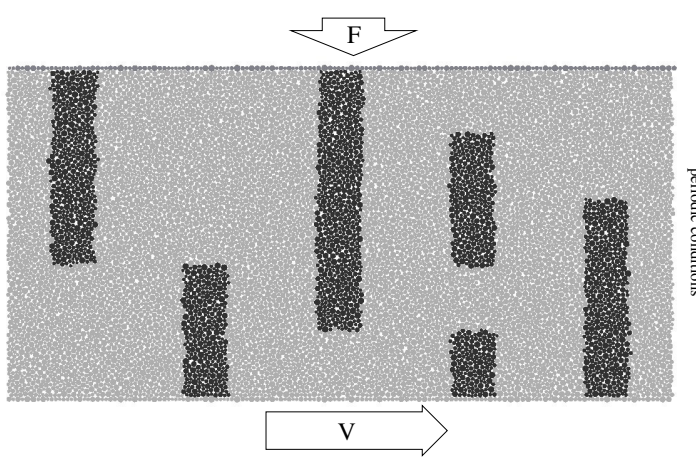

(a)

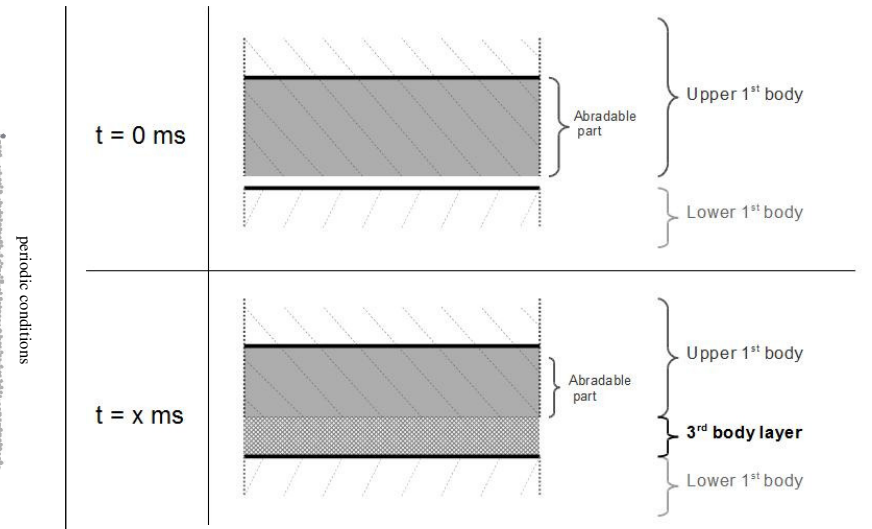

(b)

Fig. 3. (a) Granular model principle and micro-structure description: dark-gray particles represent inclusions while light-gray particles represents the matrix and (b) sketch of the model with 1st bodies (upper and lower) and 3rd bodies shown; the granular model shown in (b) is the abradable part of the upper 1 st body shown in (a)

The model is 100 micrometers long and 50 micrometers high. Particles have an average radius of 0.375 micrometers; this parameter is chosen as a compromise so that inclusions are detailed enough, yet are of the same order of magnitude as the wear particle that can be observed in friction and wear studies. The numerical parameters are summarized in table 1. 


\begin{tabular}{|c|c||c|c|}
\hline Sample length & $100 \mu \mathrm{m}$ & Computation time & $0.4 \mathrm{~ms}$ \\
Sample height & $50 \mu \mathrm{m}$ & Time step & $10^{-7} \mathrm{~ms}$ \\
Particle mean radius & $0.5 \mu \mathrm{m}$ & CZM stiffness & $10^{3}, 10^{5}, 10^{7} \mathrm{MPa}$ \\
Particle number & 10000 & Decohesion energy & $10^{-2} \mathrm{~J} / \mathrm{m}$ \\
Cohesion force & $0,10^{-4}, 10^{-3}, 10^{-2} \mathrm{~N}$ & Sliding velocity & $1 \mathrm{~m} / \mathrm{s}$ \\
& & Normal pressure & $10 \mathrm{MPa}$ \\
\hline
\end{tabular}

Table 1. Numerical parameters used in the model.

Pressure and shear velocity (i.e. tribological inputs) are applied to the model and periodic conditions are used to simulate an infinite flow. The shear velocity is constant in the simulation and equal to $1 \mathrm{~m} / \mathrm{s}$, typical of tribological processes. In the simulation, there was no velocity decay. As velocity is constant, the value of the force applied to the domain can influence on the degradation process. As the aim of this work is to understand the degradation process, the applied force (and consequently the pressure) should be high enough to produce transition phenomena. To calibrate the value of $F$, three values were tested: $0.1,1$ and $10 \mathrm{~N}$ (see Fig. 4).

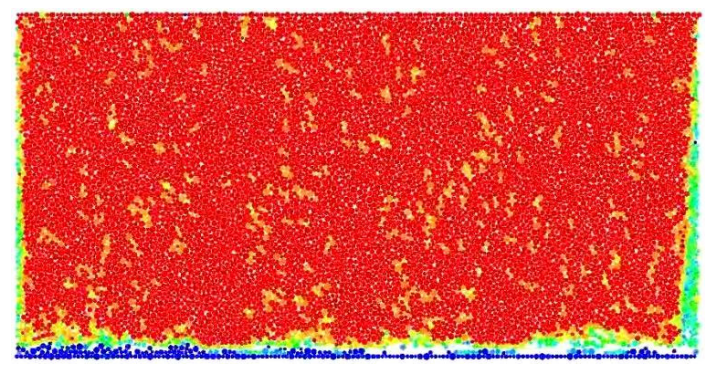

(a)

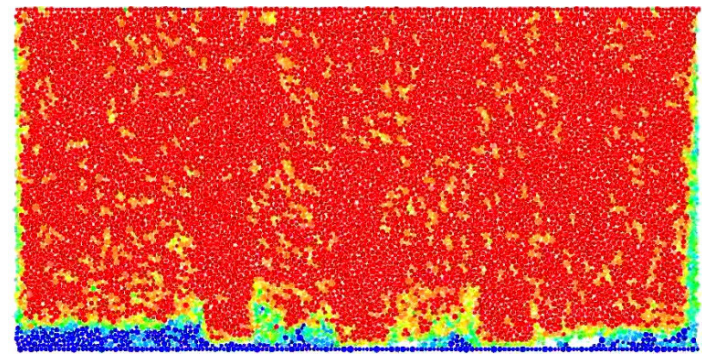

(b)

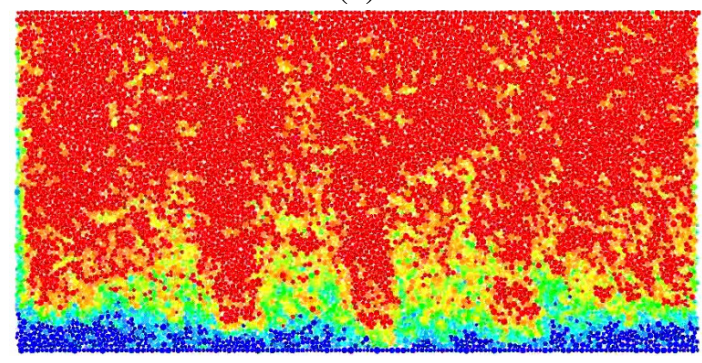

(c)

Fig. 4. Comparison between heterogeneous materials under growing normal pressure, under the same shear velocity, at the same simulation time : (a) pressure of $1 \mathrm{MPa}(0.1 \mathrm{~N}$ as normal force), (b) pressure of $10 \mathrm{MPa}(1 \mathrm{~N}),(\mathrm{c})$ pressure of $100 \mathrm{MPa}(10 \mathrm{~N})$.

As mentioned above, the range of pressure should be chosen so that the analysis of material behavior is not biased by a disproportionate pressure effect. This range is around $10 \mathrm{MPa}$ for our studies (i.e. $F=1 \mathrm{~N}$ ). Above this value, the material is crushed (see Fig. 4 c), and below, the pressure applied is not sufficient to produce any degradation in a reasonable 
computational time (see Fig. 4 a).

\subsection{Numerical measures}

To analyze the simulation results, different macroscopic quantities are used: the global damage, the macroscopic friction and the stress tensor. Each definition is given hereinafter.

\subsubsection{Global damage}

The CZM law used to model the first body is associated with a local parameter $\beta_{\alpha}\left(\alpha \in\left[1, n_{c}\right]\right)$. Using such parameter, it is possible to define two new variables.

The first, denoted $\beta_{i}\left(i \in\left[1, n_{b}\right]\right)$ is the equivalent damage of a single particle and is defined as:

$$
\beta_{i}=\frac{1}{n_{i}^{0}} \sum_{\alpha \in \mathcal{L}_{i}} \beta_{\alpha}
$$

where $n_{i}^{0}$ and $\mathcal{L}_{i}$ are the initial number of contacts and the list of contact of particles $i$, respectively. As for the parameter associated with each contact, $\beta_{i}$ ranges from 0 to 1 . If $\beta_{i}$ is equal to 1 all the contacts of the particle $i$ are preserved. If $\beta_{i}$ is equal to 0 , all its contacts are broken and the particle becomes a third-body particle.

In a similar way, the second variable defined, $\beta$, corresponds to the global damage of the sample and is defined as:

$$
\beta=\frac{1}{n^{0}} \sum_{\alpha \in \mathcal{L}} \beta_{\alpha},
$$

where $n^{0}$ and $\mathcal{L}$ are the initial number and the list of contacts in the whole sample, respectively. When plotted for a given geometry, a value of 1 (red color on the simulation snapshots) means that the sample is intact: the more the curve tends to lower values, the more the sample is damaged. To sum up, the graph represents the integrity of the sample.

\subsubsection{Global friction}

Experimentally, the friction coefficient is measured as the ratio between the force in the sliding direction and the force applied to the system. To reproduce these measurements in the simulations, the global friction coefficient $\mu(t)$ is defined as:

$$
\mu(t)=\frac{\sum_{i=1}^{n_{L B}} r_{x}^{i}(t)}{F}
$$

where $r_{x}$ is the component of the force in the sliding direction, exerted on one of the $n_{L B}$ particles composing the lower boundary, while $\mathrm{F}$ denotes the normal force applied to the upper body.

\subsubsection{Equivalent stress tensors}

In a discrete element assembly, an equivalent strain tensor may be computed [28] to reveal macroscopic quantities such as the hydrostatic pressure $p$ or the deviatoric stress $q$. The stress tensor of a particle $\mathrm{P}, \sigma(P)$, is defined as typical for granular material as [28] :

$$
\sigma_{i j}(P)=\frac{1}{\operatorname{vol}(P)} \sum_{\alpha \in \mathcal{L}_{p}} r_{i}^{\alpha} l_{j}^{\alpha},
$$

where, for two particles $i$ and $j, r^{\alpha}$ is the force exerted on the contact, $\alpha, l$ the distance between the contact point and the centre of mass of the particle $P$, and $\mathcal{L}_{p}$ the contact list of the particle $P$.

In a biphasic material, such a tensor may be computed within each phase (matrix and inclusion) and also at the matrixinclusion interface. Moreover, when dealing with first body degradation, it is also possible to perform such a computation for the first body, the third-body and for the interface between them; then, computing the value of the $q / p$ ratio can give an idea of where the relative motion is accommodated. 


\section{Results}

\subsection{Wear of homogeneous material}

First, a simulation using homogeneous models was performed in order to obtain reference results for the analysis of the degradation of biphasic materials. Two homogeneous models were created with equal CZM parameters: a value of $C_{N}$ of $10^{7}$ for homogeneous model 1 and a value of $10^{5}$ for homogeneous model 2 . A heterogeneous model combining the two values of $C_{N}$ was also created: the larger value for the interactions between inclusion particles and the lower value for the interactions between matrix particles as well as between inclusion and matrix particles. In this case, when debris particles were created, they evolved with cohesion-less interactions. The resulting damage evolution is presented in Fig. 5.

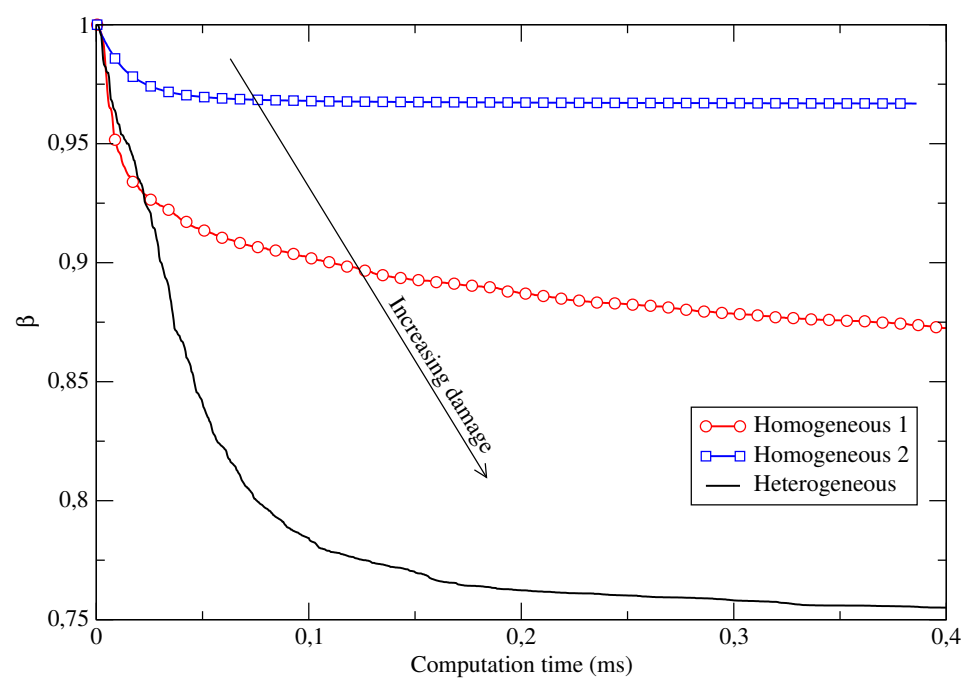

Fig. 5. Evolution of global damage for two homogeneous materials and a heterogeneous material under normal pressure and shear velocity.

Different observations may be made. First, the lower damage value was obtained for homogeneous model 1 i.e. the material with the highest CZM stiffness parameter. The highest value was not obtained for homogeneous model 2 but for the heterogeneous model. Consequently, the damage evolution of a biphasic material could not be limited to the damage evolution of a homogenous model composed of a single phase. Even if damage values are different, a stabilization of the global damage curve can be observed for all models. Damage stabilization, which occurs for each model, can easily be explained by the creation of a third-body layer which plays a protective role, accommodating the relative velocity by shearing within itself (see Fig. 6).

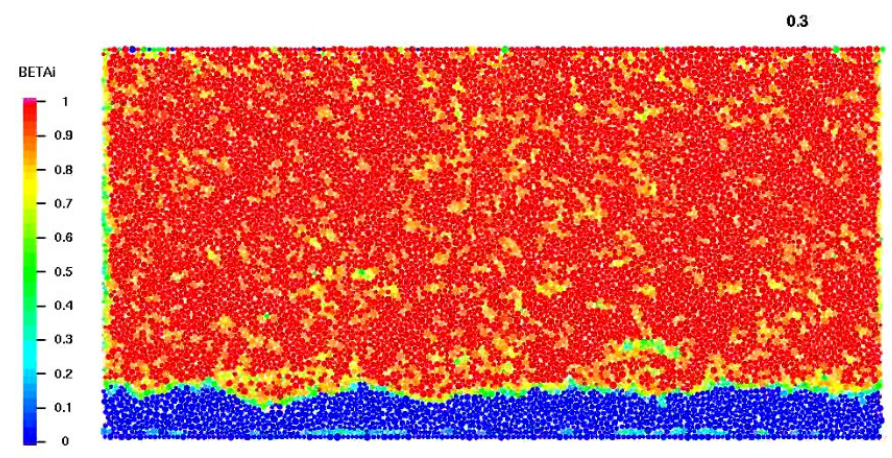

Fig. 6. Steady state of a homogeneous material submitted to tribological solicitations with the establishment of a protective third-body layer (seen at the bottom of the model).

This phenomenon is possible because of the presence of periodic conditions, representing an infinite contact where all debris particles stay in the contact, whereas in a "real" mechanism the contact can retain more or less particles depending on 
the evolution of the whole system (first body vibrations, separation, acceleration,...). The role and influence of this third-body layer will be discussed more precisely later on.

\subsection{Wear of heterogeneous material}

In order to study the influence of inclusion location and length, different microstructures were created (see Fig. 7), all with the same ratio between inclusion surface and matrix surface. Three heterogeneous models were used, characterized by different inclusion lengths, denoted by 1 , reaching the frictional surface: mixed inclusions, short inclusions $(l<H / 2)$, long inclusions $l<H / 2$, where $H$ is the height of the sample.

(a)

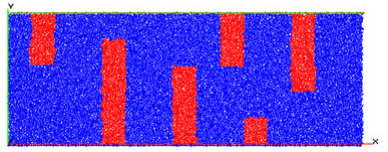

(b)

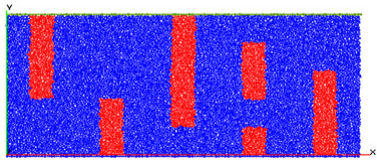

(c)

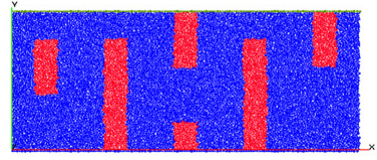

Fig. 7. Geometry of the three heterogeneous models used, characterized by different properties of inclusions reaching the frictional surface: (a) mixed inclusions, (b) short inclusions, (c) long inclusions.

In each case, the stiffness of the matrix is always smaller than the stiffness of the inclusions. The same inputs were applied to each model: a pressure of $10 \mathrm{MPa}$ and a sliding velocity of $1 \mathrm{~m} / \mathrm{s}$. The normal stiffness $C_{N}$ has a value of $10^{7}$ for the interactions between inclusion particles and a value of $10^{5}$ for the interactions between matrix particles as well as between inclusion particles and matrix particles. To activate damage faster, cohesion was allowed between the moving first body (lower body) and all the particles which constitute the upper degradable first body. With these tribological inputs, the damage in each model evolved in the same way (a transitory stage and a steady stage) but to a different final value of damage for each case (see Fig. 8).

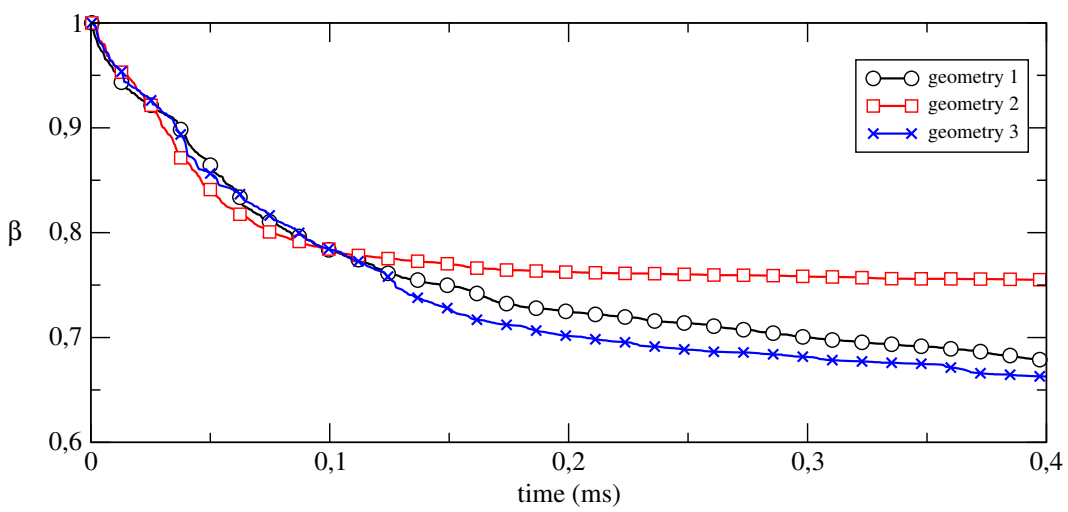

Fig. 8. Evolution of global damage of the three heterogeneous models.

To learn more about the damage mechanisms in each model, the mechanism of third-body layer creation and composition was investigated, just as in real life contact. Fig. 9 shows the global damage of each phase in each model: matrix/matrix, inclusion/matrix and inclusion/inclusion.

The results clearly show that the percentage of broken contacts is larger for the matrix/matrix interactions than for the matrix/inclusion or inclusion/inclusion interactions. Indeed the overall magnitude of broken matrix/inclusion contacts is also relevant (around 25\% of contacts are broken). The inclusion/inclusion contacts are much less affected (the maximum damage observed is close to $2.5 \%$ ). The damage of each phase occurs mainly during the first quarter of the simulation time. Then, once all contacts are broken, debris particles evolve with cohesion-less interactions (just as in the homogeneous models), creating a "fluid-like" layer which easily accommodates the relative motion between first bodies. This film prevents upper first body degradation and is responsible for the steady state observed in the global damage graphs. The action of the layer is reinforced by the periodic boundaries which allow all the particles to be retained in the contact, maintaining a continuous internal flow of particles once the layer is established. 
(a) matrix/matrix

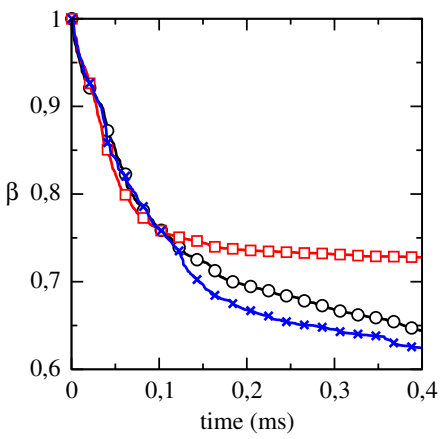

(b) inclusion/matrix

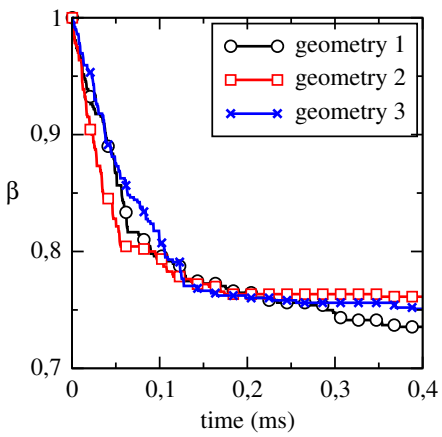

(c) inclusion/inclusion

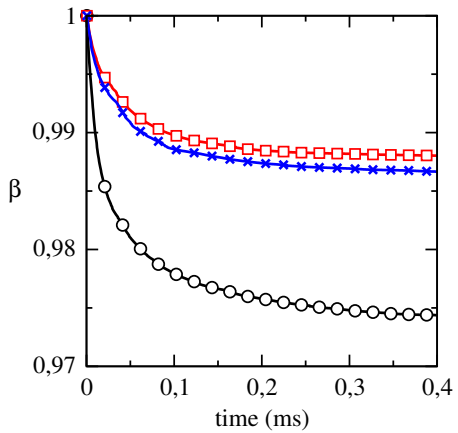

Fig. 9. Evolution of global damage of the three heterogeneous models in each phase of the material : (a) matrix 1, (b) inclusion/matrix and (c) inclusions.

(a)

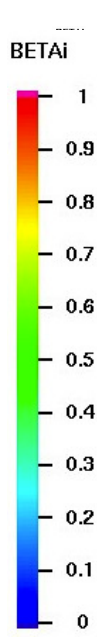

0.025

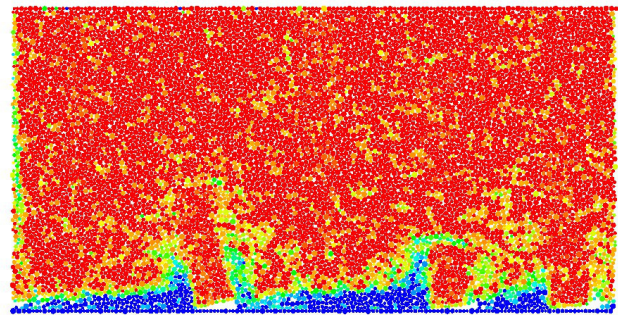

(c)

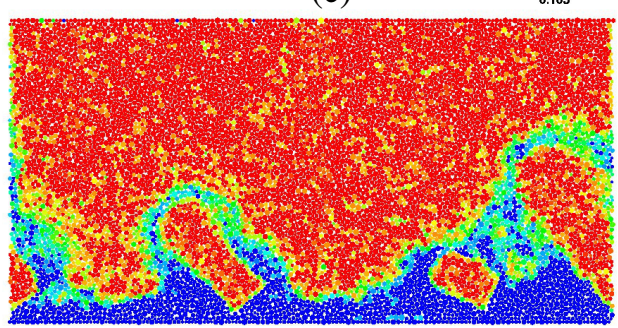

(b)

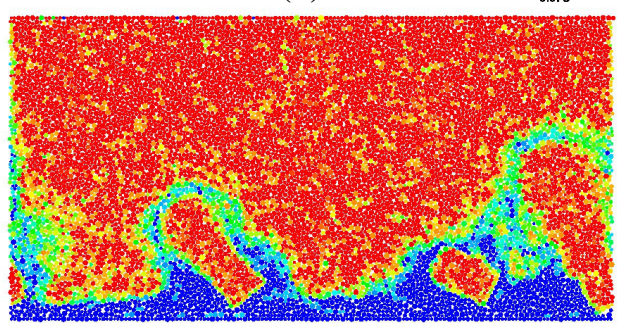

(d)

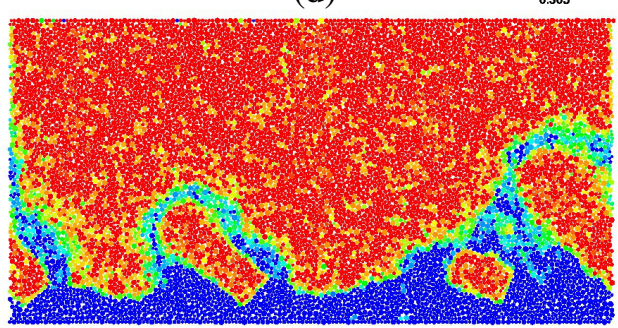

Fig. 10. Visualisation of the evolution of $\beta_{i}$ during the tribological process for the geometry 2 at time: (a) $t=0.025$, (b) $t=0.075$, (c) $t=0.10$ and (d) $t=0.3$.

Figure 10 presents the aspect of material (microstructure 2) during the tribological process. During the transient phase $(t<0.1$, see Fig. 9 and Figs. 10a,b,c) the matrix damage mainly appears where inclusions reach the friction surface, where matrix particles contribute, step by step, to the formation of the third-body layer. A comparison of Figs. 10c and Figs. 10d shows that the third-body layer, once established, has a "protective" role which explains the steady state observed in Fig. 9 : the process which damages the material is stopped.

Keeping this phenomenon in mind, it can easily be seen that to reduce the amount of global damage, the microstructure should allow the creation of a third-body layer, easily and quickly. In heterogeneous microstructures, stiff inclusions have a large influence: if they are too long or not mobile enough to allow the circulation of debris particles, the creation of the third-body layer is disturbed. Thus, accommodation still occurs in the first body volume which continues to be deformed. Microstructures with longer inclusions reaching the friction surface (microstructure 3), show the greatest final damage value (see Fig. 8). In Fig. 9, it can be seen that each microstructure contains the same relative amount of broken inclusion/matrix links. Yet whilst this amount is sufficient, with microstructure 2, to allow the creation of a third-body layer and to stabilize its damage (stabilization of the number of intact matrix/matrix links ), this is not the case for the others. When the circulation of debris particles is disturbed, there occurs a so-called "corner effect" between the remaining matrix and the inclusions: the matrix degrades until the inclusions become more mobile. Damage continues to accumulate (transient state) until the inclusions gain sufficient mobility to allow particles to circulate freely enough for the third body layer to be established; both damage and friction then stabilize (steady state).

Similar observations may be made of the macroscopic friction. Figure 11 presents the evolution of the $q / p$ ratio for the first and the third-body, compared to the global friction coefficient (see Eq. 9). During the transient phase, the friction 
coefficient value is closer to the $\mathrm{q} / \mathrm{p}$ ratio of the first body, underlining the fact that accommodation takes place mostly within the first body. The ratio then gradually falls to reach a value close to the $q / p$ ratio of the third-body, showing that accommodation takes place within the third-body layer during the steady state.

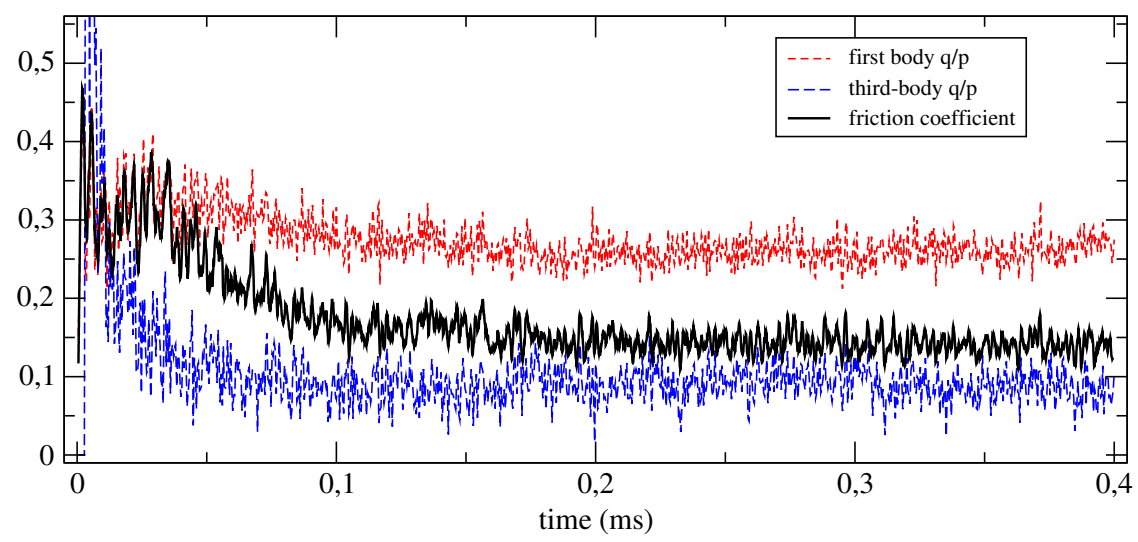

Fig. 11. $q / p$ values of the equivalent strain tensors of 1 st and $3 r d$ body, compared to global friction coefficient.

To understand the impact of interaction forces on the distribution of inclusion geometries, simulations were performed for the three microstructures with constant $C_{N}$ values for interactions between inclusion particles (equal to $10^{7}$ ) and for interactions between matrix particles (equal to $10^{5}$ ). The value of the interaction between inclusion particles and matrix particles takes the value of $10^{3}, 10^{5}$ and $10^{7}$. The results of the evolution of $\beta$ are presented respectively in Fig. 12a, Fig. $12 \mathrm{~b}$ and Fig. 12c.

(a)

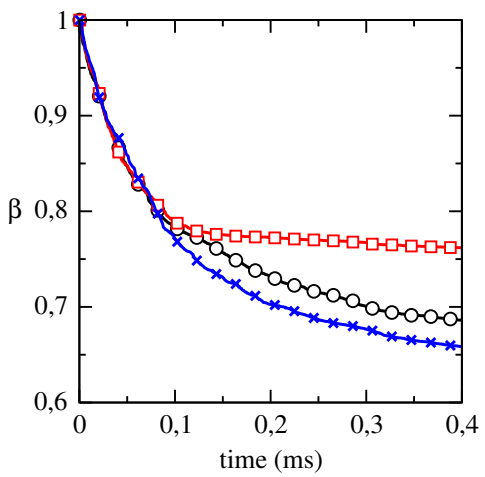

(b)

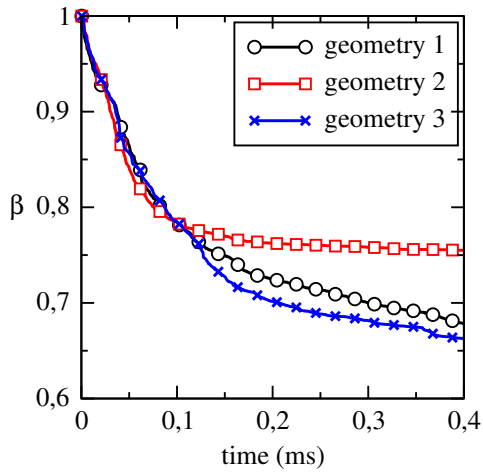

(c)

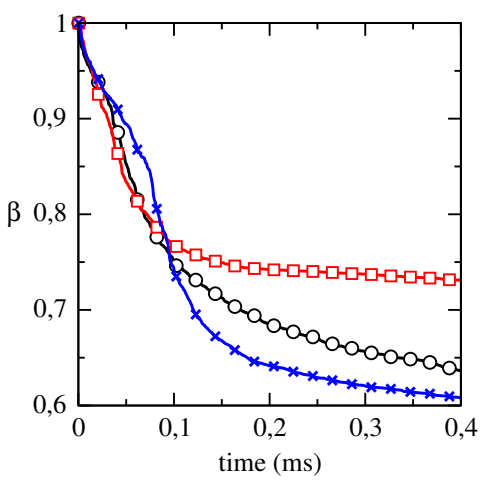

Fig. 12. Evolution of the global damage $\beta$ for value of the interaction between inclusion particles and matrix particles of (a) $10^{3}$, (b) $10^{5}$ and (c) $10^{7}$

If there is some variation in the evolution of $\beta$ in the first part of the simulations, the microstructures are always ranked the same $\left(\beta_{G 2}<\beta_{G 1}<\beta_{G 3}\right)$ at the end of the simulated process (c.f. Fig. 12). This shows that the inclusion shape and distribution is more important than the inclusion/matrix interface strength. The greater this strength, the greater the damage. The inclusion/matrix interactions limit the damage in the structure like the fuse in an electrical circuit. If the threshold value is too large, the high rigidity of inclusions directly affects the matrix, favoring its degradation (stress and deformation being transmitted from inclusions to matrix). When the threshold value is small, the interface quickly breaks down, and the inclusions are detached from the matrix allowing more accommodation at lower degradation. The impact of this "fuse effect" is also observed in the evolution of the global friction $\mu$ (c.f. Fig. 13).

When interaction between inclusion particles and matrix particles is high, the global friction remains high for longer, except for microstructure 2 whose global friction and damage are not so much affected by the different threshold values.

However, Fig. 13 shows that the friction coefficient always has the same final value during its steady regime, whatever the model. This is easily explained by the fact that during the steady state, friction is controlled by the shearing of the 
(a)

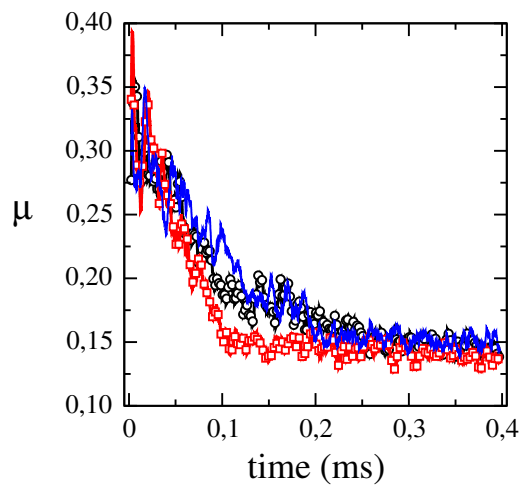

(b)

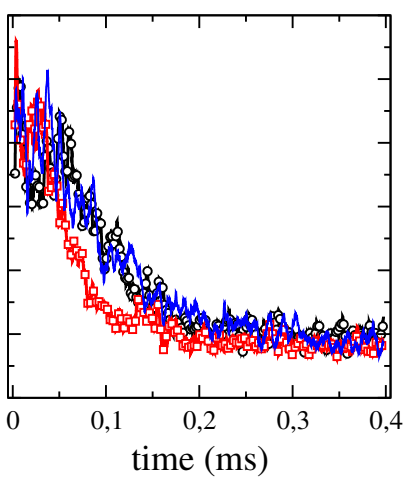

(c)

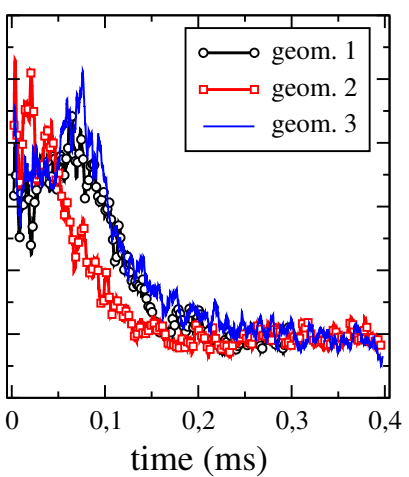

Fig. 13. Evolution of the global friction $\mu$ for the value of the interaction between inclusion particle and matrix particles of (a) $10^{3}$, (b) $10^{5}$ and (c) $10^{7}$

third-body layer alone (where, as shown above, all relative velocity is accommodated). The media and interaction laws for this layer are the same in all models which therefore show the same global friction coefficient; a possible improvement of the models is then to introduce adhesive forces within this medium, and this is considered in the next section.

\subsection{Wear of heterogeneous material with adhesive third-body}

Physico-chemical aspects are known to be very important in tribological phenomena [23, 31]. Particles are always reactive, and this should be represented in a numerical model. To understand the influence of such reactivity on the damage process, three levels of cohesive forces were tested. The cohesive force intervenes in the media once CZM links are broken (i.e. $\beta_{\alpha}=0$ ). The values of cohesive force chosen were $10^{-4}, 10^{-3}, 10^{-2}$ (common values for this particle size and external forces) and will be called "weak", "medium" and "strong" forces, respectively. The cohesion for the contact between the two first bodies is the same as between the debris particles.

Figure 14 presents the evolution of the global damage $\beta$ with the different cohesion values (i.e. $10^{-4}, 10^{-3}$ and $10^{-2}$ ) for model 2. The results are compared to a full cohesion-less simulation (no cohesion within third-body particles and between particles and the lower first body) and the previous case (no cohesion within third-body particles and a cohesion of $10^{-2}$ between particles and the lower first body).

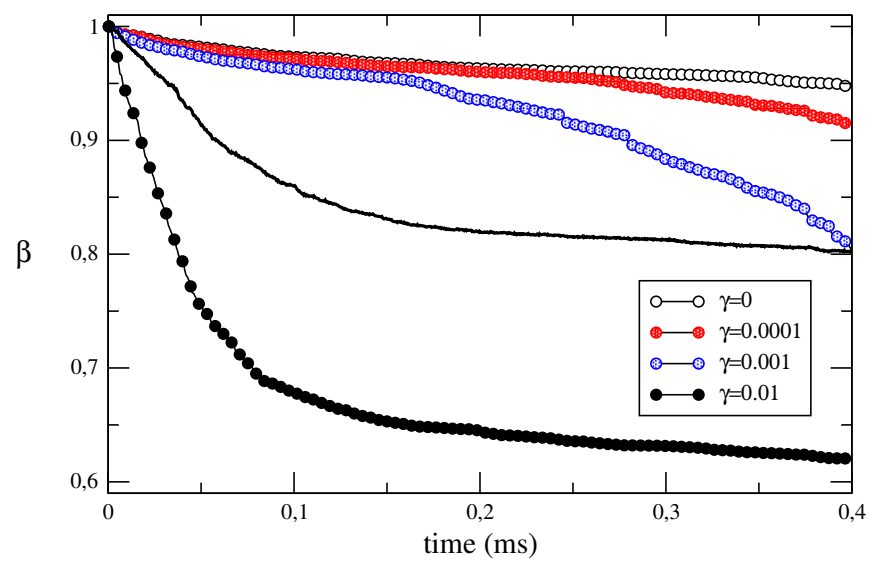

Fig. 14. Global damage with different level of adhesion force in the third-body layer. The plain line correspond to the result of the previous section.

The evolution of the global damage is strongly dependent on the cohesion between the detached particles. For a given simulated time, a larger cohesion value leads to greater global damage. However, with strong cohesion, the global damage reaches a quasi steady state; yet that is not the case with lower cohesion. In this case the damage process seems to start and continue for a time longer than the run time of $0.4 \mathrm{~ms}$. Consequently, it is difficult to give a direct correlation between global 
damage and the cohesion value. It can also be noted that the example discussed in the previous section with no cohesion between third-body particles and a cohesion of $10^{-2}$ between particles and the lower first body was more damaged than the full cohesion-less example. This underlines the influence of the initial cohesion between the first bodies. If the cohesive properties of the debris particles are important, the initial surface reactivity has also much influence on the damage process.

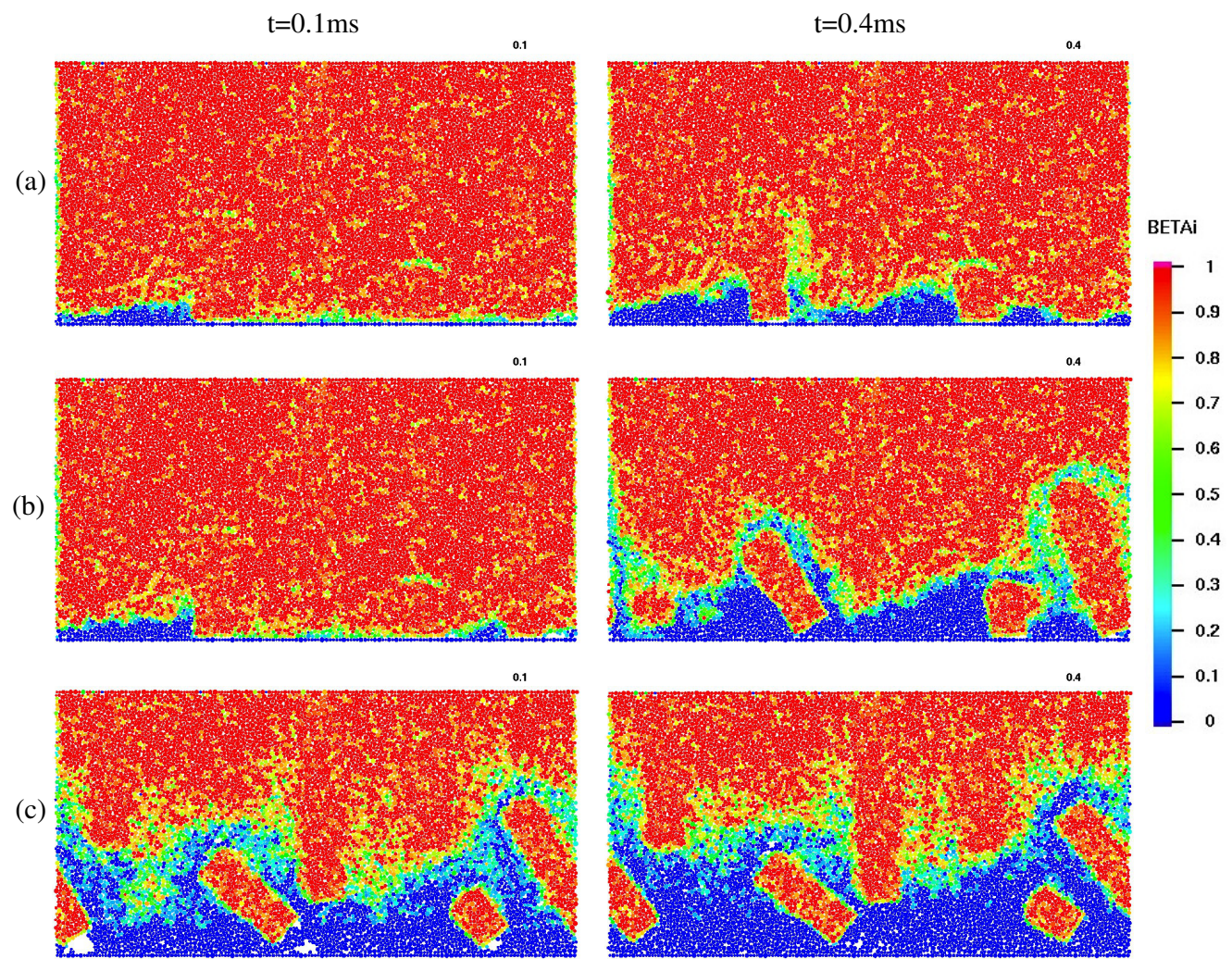

Fig. 15. Aspect at time $t=0.1 \mathrm{~ms}$ and $t=0.4 \mathrm{~ms}$ of damaged material (geometry 2) for a weak (a), medium (b) and strong (c) cohesion value for debris particles.

Figure 15 shows the appearance of microstructure 2 with the weak (a), medium (b) and strong (c) cohesion value for debris particles after $0.1 \mathrm{~ms}$ and at the end of the simulation. Such visualizations corroborate the comments concerning Fig. 15. For the strong cohesion (case (c)), snapshots at the chosen times are quasi identical, in terms of degradation and deformation. This is not the case for the weak and medium cohesion values where there are numerous differences between the snapshots at different times. It is interesting to note that Fig. $15 \mathrm{a}$ at $t=0.4$ resembles Fig. $15 \mathrm{~b}$ at time $t=0.1$. This shows the need for longer simulations to determine whether the cohesion is simply an accelerator of the damage process (same damage level for different times). However, the global damage level is higher with strong adhesion forces, due to adhesive wear; thus 3rd body layer thickness is greater with high cohesion (debris particles being always in contact) than without cohesion.

Figure 16 proposes an interpretation of the influence of the debris particle cohesion in terms of global friction evolution, for the case of microstructure 2. For strong cohesion, the global friction is nearly constant during the simulation process (the transitory stage being very short), while for the weak and medium cohesion values, it increases during the process, showing that no steady state has been reached. It can also be noted that the sample used in the previous section presents an initial friction value close to the value for strong cohesion and then decreases quickly to the value of the full cohesion-less simulation. If the initial surface reactivity leads to a large friction value (as well as a large damage gradient), the third-body layer flow could subsequently become dominant in the frictional process.

The results for other microstructures (see Fig. 17) show that when the adhesion parameter is high enough to allow the microstructure to reach a steady state, inclusion distribution and length have the same effects as without adhesion $\left(\beta_{G 2}<\right.$ $\left.\beta_{G 1}<\beta_{G 3}\right)$. The mechanism of accommodation in the 3rd body layer still occurs, and microstructures that allow the easy creation of the layer still show the least damage. The effects of other parameters, as mentioned above, require longer simulations to confirm that the trends are the same. To conclude, it can be said that with adhesion, different levels of 


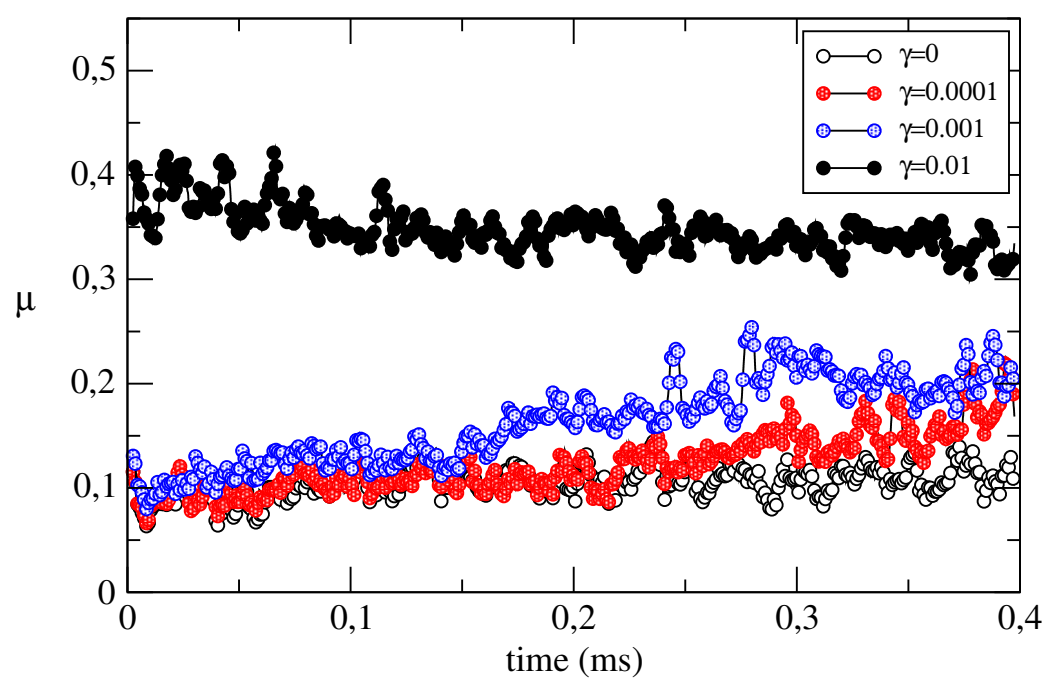

Fig. 16. Friction coefficient of the geometry 2 with different level of adhesion force in the 3rd body layer.

(a) $g=0.0100$

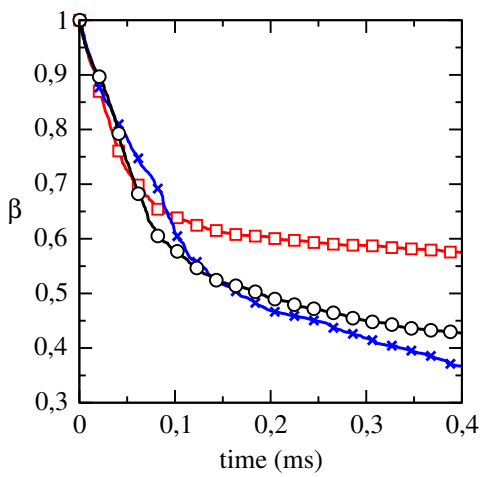

(b) $\mathrm{g}=0.0010$

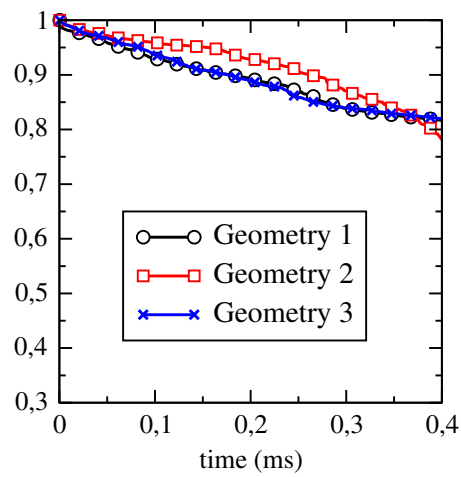

(c) $\mathrm{g}=0.0001$

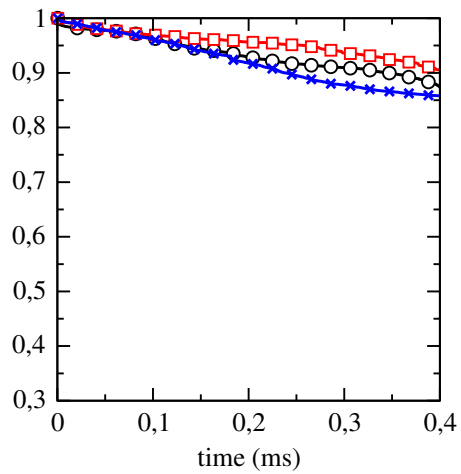

Fig. 17. Global damage of the different geometries, with different levels of adhesion force in the third-body layer : (a) $g=0.0100$, (b) $g=0.0010$, (c) $g=0.0001$

damage and friction coefficient arise, giving an "environmental parameter" which affects the wear of a microstructure without modifying the general behavior that depends on its geometrical characteristics.

\section{Discussion}

Some of the phenomena predicted here can be found in experimental studies, particularly those studying heterogeneous friction materials. In [22], the authors study the friction of brake pad material; Fig. 18 shows a SEM-micrograph and a sketch of the friction interface of such materials, with heterogeneities reaching that surface. The authors show that the 3rd body layer is "mainly responsible for the friction response", and that the nature and quantity of particles retained in the contact influence the formation of this film. The same observation is made for homogeneous materials, but in heterogeneous materials the friction surface is obviously particular. Wear troughs that appear at the surface, influenced by the presence of micro-structure and inclusions reaching the surface, play an important role in facilitating or hampering the circulation of particles in the contact, influencing the behavior of the 3rd body layer and thus influencing the friction and wear behavior of the whole contact. The nature of the particles is also significant: they may come from the matrix or from inclusions which do not have the same (mechanical, chemical...) properties. The same kind of observations are made for other heterogeneous friction materials, such as Carbon/Carbon friction composites (see Fig. 19). In such materials, micro-structure and porosity influence the life of the 3rd body in the contact. For example they may allow "particle pools" to form which remove the "dangerous" character of the "corner effect" discussed above, by helping to feed the third-body layer [23]. The numerical results given above, use internal cohesions of 0 and $10^{-2}$ which are not representative of such a material: The first leads to a powdery third-body and the second to an abrasive third-body. One could imagine that, according to the physical properties of the interface, the cohesion value may evolve during the sliding process, but this idea needs experimental verification. 


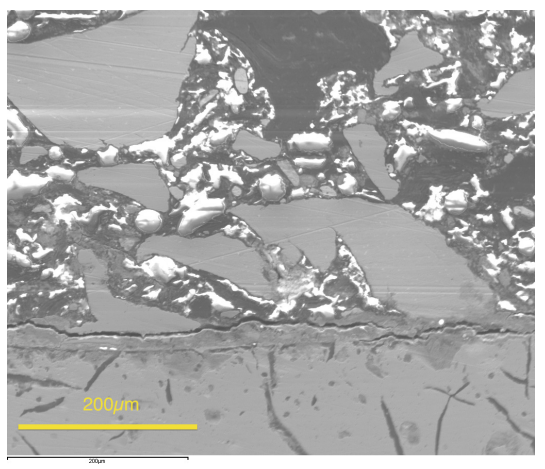

(a)

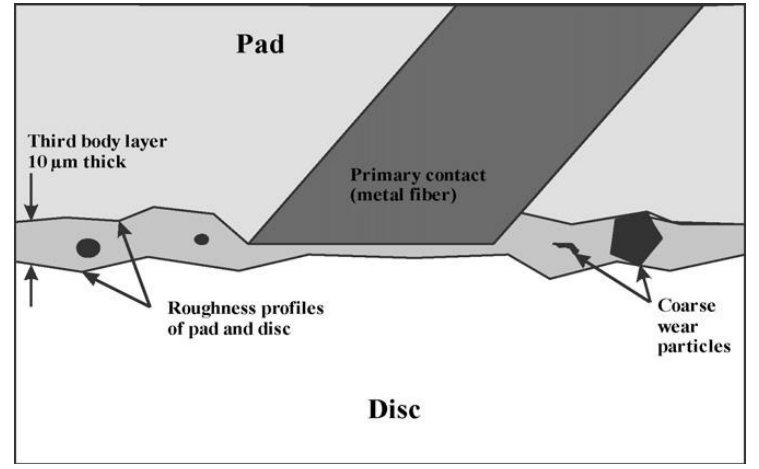

(b)

Fig. 18. Interface between brake pad friction materials, (a) SEM-micrograph and (b) sketch (courtesy of W. Oesterle [22])

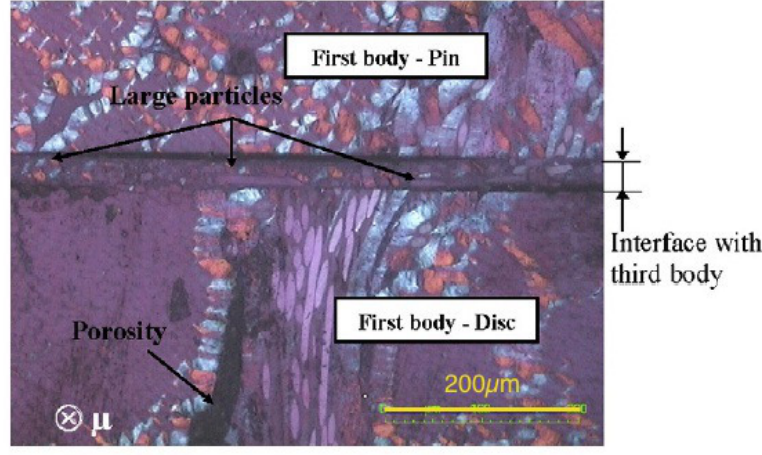

(a)

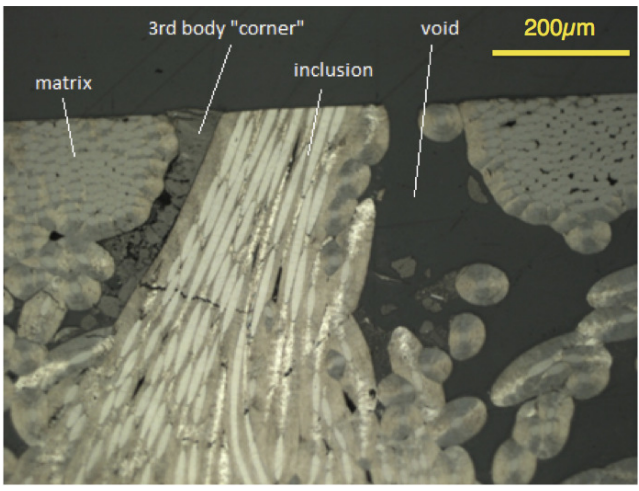

(b)

Fig. 19. (a) Interface between Carbon/Carbon friction materials, SEM-micrograph (courtesy of $\mathrm{H}$. Kasem [23]) and (b) Optical microscopy cross section view of CC composite after friction showing heterogeneous aspect and 3rd body "corner effect"

To conclude, numerical models like those presented in this study may be useful for understanding heterogeneous material behavior in terms of friction and wear, depending on micro-structure geometrical characteristics, material mechanical properties and interactions occurring in the 3rd body layer. The use of the Discrete Element Method to model the wear of a material is not an innovation in itself. However, the use of the NSCD method with CZM and adhesive laws gives very interesting advantages, of which the most important is to model the evolution of damage throughout the life of the contact. Every aspect of a tribological problem at the local scale can be taken into account, even if temperature and thermo-mechanical aspects are still to be studied. More important, the predicted local friction coefficient and wear are key parameters which could be used in higher scale finite element models, replacing the arbitrary, and sometimes meaningless, parameters currently used. All in all, the model is a numerical tool of experimental investigation. Apart from the consideration of temperature, other improvements could be made to overcome the limitations highlighted earlier in this paper. First of all, an upscaling of the models will be made, with bigger models and comparatively smaller inclusions. This should lead to a reduction in the bias due to the "scale effect", and should allow other material parameters, such as the volume ratio of inclusions, to be studied more precisely. 3D models will also be built, with cylindrical inclusions, in order to avoid the so called "scraping effect" and to introduce a better description of mechanical systems. The introduction of a "wear flow" with evacuation of debris particles outside the contact is also planned.

\section{Acknowledgements}

Part of this work is funded by the Agence Nationale de la Recherche in the framework of the ANR-10-RMNP-0013 project. 


\section{References}

[1] Berthier, Y., 2001. Handbook of Materials Behavior models. Lemaître Academic Press, ch. Background on friction and wear, pp. 676-699.

[2] Archard, J. F., 1953. "Contact and rubbing of flat surfaces". J. Appl. Phys., 24(8), pp. 981-988.

[3] Rhee, S., 1970. "Wear equation for polymers sliding against metal surfaces". Wear, 16(6), pp. 431-445.

[4] Barwell, T., 1957. "Wear of metals". Wear, 1, pp. 317-322.

[5] Chongyi, C., Chengguo, W., and Ying, J., 2010. "Study on numerical method to predict wheel/rail profile evolution due to wear". Wear, 269, pp. 167-173.

[6] Lee, J., Xu, G., and Liang, H., 2001. "Experimental and numerical analysis of friction and wear behaviour of polycarbonate". Wear, 251(1-12), pp. 1541-1556.

[7] Agelet de Saracibar, C., 1999. "On the numerical meodeling of frictional wear phenomena". Comput. Methods Appl. Mech. Engrg., 177, pp. 401-426.

[8] Torrance, A., 2005. "A method for calculating boundary friction and wear". Wear, 258(5-6), pp. 924-934.

[9] AbuBakar, A., and Ouyang, H., 2008. "Wear prediction of friction material and brake squeal using the finite element method". Wear, 264(11-12), pp. 1069-1076.

[10] Gallego, L., Fulleringer, B., Deyber, S., and Nélias, D., 2010. "Multiscale computation of fretting wear at the blade/disk interface". Tribol. Int., 43(4), pp. 708-718.

[11] Gallego, L., Nélias, D., and Deyber, S., 2010. "A fast and efficient contact algorithm for fretting problems applied to fretting modes i, ii and iii”. Wear, 268(1-2), pp. 208-222.

[12] Jourdan, F., 2010. "A numerical model for multidirectional wear". Wear, 268, pp. 1380-1386.

[13] Berthier, Y., Godet, M., and Brendle, M., 1989. "Velocity accomodation in friction". STLE Tribology T., 32(4), pp. 490-496.

[14] Sun, Y., 1990. "Etude mcanique des couches minces en tribologie: une approche numrique". PhD thesis, INSA Lyon.

[15] Higgs, C., and Tichy, J., 2004. "Granular flow lubrication : continuum modeling of shear behaviour". ASME J. Tribol., 126, pp. 499-509.

[16] Muller, M., and Ostermeyer, G., 2007. "A cellular automaton model to describe the three-diemnsional friction and wear mechanism of brake systems". Wear, 263, pp. 1175-1188.

[17] Dimitriev, A., Osterle, W., and Kloss, H., 2008. "Numerical simulation of typical contact situations of brake friction materials". Tribol. Int., 41, pp. 1-8.

[18] Dmitriev, A., Smolin, A., Popov, V., and Psakhie, S., 2009. "A multilevel simulation of friction and wear by numerical methods of discrete mechanics and a phenomenological theory". Phys. Mesomech., 12(3-4), pp. 11-19.

[19] Fillot, N., Iordanoff, I., and Berthier, Y., 2004. "A granular dynamic model for the degradation of material". ASME J. Tribol., 126(3), pp. 606-614.

[20] Renouf, M., Saulot, A., and Berthier, Y., 2006. "Third body flow during a wheel/rail interaction". In 3rd European conference on computational mechanics solids, M. S. CA, ed.

[21] Godet, M., 1984. "The third-body approach: a mechanical view of wear". Wear, 100(1-3), pp. 437-452.

[22] Osterle, W., Dorfel, I., Prietzel, C., Roocha, H., A.-L., C.-B., Degallaix, G., and Desplanques, Y., 2009. "A comprehensive microscopic study of third body formation at the interface between a brake pad and brake disc during the final stage of a pin-on-disc test”. Wear, 267, pp. 781-789.

[23] Kasem, H., Bonnamy, S., Rousseau, B., Estrade-Szwackopf, H., Berthier, Y., and Jacquemard, P., 2007. "Interdependence between wear process, size of detached particles and co2 production during carbon/carbon composite friction". Wear, 263, pp. 1220-1229.

[24] Elrod, H., and Brewe, D., 1992. "Numerical experiments with flows of elongated granules". Tribology S., 21, pp. 219226.

[25] Jerier, J., and Molinari, J., 2012. "Normal contact between rough surfaces by the discrete element method". Tribol. Int., 47(0), pp. 1-8.

[26] Moreau, J. J., 1988. "Unilateral contact and dry friction in finite freedom dynamics". In Non Smooth Mechanics and Applications, CISM Courses and Lectures, J. Moreau and e. P.-D. Panagiotopoulos, eds., Vol. 302 (Springer-Verlag, Wien, New York), pp. 1-82.

[27] Jean, M., 1999. "The non smooth contact dynamics method". Compt. Methods Appl. Math. Engrg., 177, pp. 235-257.

[28] Radjai, F., Jean, M., Moreau, J.-J., and Roux, S., 1996. "Force distributions in dense two-dimensional granular systems". Phys. Rev. Lett., 77(2), pp. 264-277.

[29] Cao, H.-P., Renouf, M., Dubois, F., and Berthier, Y., 2011. "Coupling continuous and discontinuous descriptions to model first body deformation in third body flows". ASME J. Tribol., 133(4), p. 041601.

[30] Renouf, M., and Berthier, Y., 2011. Discrete numerical modeling of granular materials. Lavoisier, ch. Numerical modeling of heat production and transmission, pp. 381-400.

[31] Renouf, M., Cao, H.-P., and Nhu, V.-H., 2011. "Multiphysical modeling of third-body rheology". Tribol. Int., 44(4), pp. 417-425. 
[32] Renouf, M., Dubois, F., and Alart, P., 2004. "A parallel version of the Non Smooth Contact Dynamics algorithm applied to the simulation of granular media". J. Comput. Appl. Math., 168, pp. 375-38.

[33] Elices, M., Guinea, G., Gómez, J., and Planas, J., 2002. "The cohesive zone model : advantages, limitations and challenges". Eng. Fract. Mech., 69(2), pp. 137-163.

[34] De Borst, R., 2003. "Numerical aspects of cohesive-zone models". Eng. Fract. Mech., 70, pp. 1743-1757.

[35] Elices, M., Rocco, C., and Rosello, C., 2009. "Cohesive cracks modelling of a simple concrete : experimental and numerical results". Eng. Fract. Mech., 76, pp. 1398-1410.

[36] Pérales, F., Bourgeois, S., Chrysochoos, A., and Monerie, Y., 2008. "Two field multibody method for periodic homogenization in fracture mechanics of nonlinear heterogeneous materials". Eng. Fract. Mech., 75(11), pp. 3378-3398.

[37] Raous, M., Cangémi, M., and Cocu, M., 1999. "A consistent model coupling adhesion, friction, and unilateral contact". Comput. Methods Appl. Mech. Engrg., 177(3-4), pp. 383-399.

[38] Frémond, M., 1987. "Adhérence des solides". J. Mec. Theor. Appl., 6, pp. 383-407.

[39] Rognon, P., Roux, J.-N., Naaim, M., and Chevoir, F., 2008. "Dense flows of cohesive granular materials". Journal of Fluid Mechanics, 596(1), pp. 21-47. 